\title{
Is there an assignment of top and bottom during symmetry perception?
}

\author{
Johan Hulleman, Glyn W Humphreys \\ Behavioural Brain Sciences, School of Psychology, University of Birmingham, Birmingham B15 2TT, \\ UK; e-mail: j.hulleman@bham.ac.uk
}

Received 14 May 2003, in revised form 31 October 2003

\begin{abstract}
We report data from a discrimination task in which participants had to decide whether a single-item display was either vertically symmetric or asymmetric. This decision was found to be easier when items with a top-bottom polarity were placed in an orientation where the wide end was the base of the stimulus and the narrow end was the top. The difference in reaction times between this orientation and its inversion was about $10 \mathrm{~ms}$. We suggest that top and bottom labels are assigned to stimuli during the detection of bilateral symmetry.
\end{abstract}

\section{Introduction}

Over the past century, there has been a considerable amount of interest in the perception of symmetry, both from the field of cognitive psychology (for a review see Wagemans 1995), and in image processing and computational theory (eg Dakin and Watt 1994; Osorio 1996). Among the issues that have been studied in some depth in cognitive psychology is the influence on the ease of symmetry detection of factors such as the orientation of the symmetry axis, the presence of multiple axes of symmetry, and the use of attention.

Symmetry detection is facilitated if there is a vertical axis of bilateral symmetry, and if there are multiple axes of symmetry (Palmer and Hemenway 1978). In singleitem displays, there is no influence of the number of parts on either side of the axis, suggesting that symmetry is computed in parallel across a stimulus (Baylis and Driver 1994). However, Olivers and Van der Helm (1998) have shown that, in multiple-item displays, searching for a symmetrical target yields steep search slopes. This suggests that computing symmetry is attentionally demanding.

In the present study, we provide evidence for a new factor that influences symmetry judgments: the top-bottom polarity of the shapes. Bilateral symmetry judgments are faster if shapes have a wide segment as their lowest feature and a narrow segment as their highest feature (wide base), relative to when this situation is reversed (wide top). We discuss the evidence in terms of the interaction between generic visual processing and symmetry perception.

We present two experiments. Experiment 1 was originally designed as a control experiment for another series of studies on figure-ground assignment and involved presenting a single figure with a clear figure-ground definition. We demonstrate an effect of top-bottom polarity, but it was small in magnitude. This could be because the ease of the task limited the magnitude of any effect. In experiment 2, we replicated the study, with the different shapes appearing at identical positions in the field. Despite the small size of the effect (around $10 \mathrm{~ms}$ ), the data from experiment 1 were replicated. Taken together, the data indicate a replicable influence of top-bottom polarity on symmetry detection. 


\section{Experiment 1}

\subsection{Method}

2.1.1 Participants. Ten participants (eight female, two male) were recruited, either in return for course credit or for a small payment. All participants had normal or corrected-to-normal vision, and were unaware of the purpose of this experiment.

2.1.2 Apparatus. The experiments were controlled by a Pentium III PC. The stimuli were presented on a 17 -inch VGA monitor, in $800 \times 600$ graphics mode.

2.1.3 Stimuli. The participants looked unrestrained at the stimuli (see figure 1a for examples) from standard viewing distance (around $50 \mathrm{~cm}$ ). The stimuli were presented in either black or white, on a grey background covering the whole screen. They could appear in eight different locations, within four for the wide-base versions (marked ' $\mathrm{B}$ ' in figure 1b) and four for the wide-top versions (marked ' $\mathrm{T}$ ' in figure 1b), within a virtual horizontal bar $(3.7 \mathrm{deg} \times 12.1 \mathrm{deg})$, presented at the centre of the screen. The vertical size of a stimulus was $3.7 \mathrm{deg}$. Each stimulus was composed of two halves, and each half consisted of 16 arms, each arm having a vertical thickness of $0.2 \mathrm{deg}$. The maximum width of an arm was $1.1 \mathrm{deg}$. The minimum width was $0.2 \mathrm{deg}$. The widths of 8 of the arms were chosen randomly to be between the minimum and the maximum width. The widths of the other 8 corresponding arms were chosen in such a way that the total sum of the arm widths was $1.4 \mathrm{deg}$.
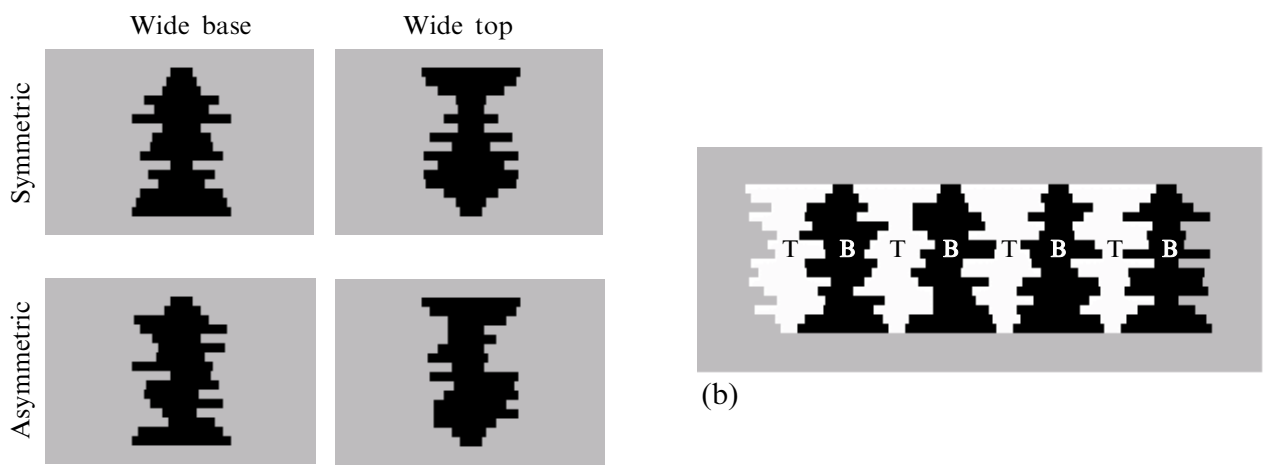

(b)

(a)

Figure 1. (a) Examples of stimuli used in experiments 1 and 2. Top left panel: symmetric, wide base; top right panel: symmetric, wide top; bottom left panel: asymmetric, wide base; bottom right panel: asymmetric, wide top. (b) Locations in which the stimuli were presented in experiment 1. Wide-top stimuli were presented in the locations marked with ' $T$ ', wide-base stimuli in the locations marked with 'B'. In experiment 2 , the locations for wide-top and wide-base stimuli were superimposed.

One set of stimuli (wide base) was constructed so that the arm at the bottom end had the maximum width, whereas the corresponding arm at the top end had the minimum width. In addition, the second arm from the bottom end was constrained to be near the maximum width, and, correspondingly, the second arm from the top was near the minimum width. A second set of stimuli (wide top) were simply inversions of the first set. Complete stimuli were created by combining two halves. So the maximum width of an entire stimulus was $2.3 \mathrm{deg}$, the minimum width $0.5 \mathrm{deg}$. Asymmetric shapes used two independent halves, symmetric shapes used the same half twice.

In a pilot experiment, twenty-two participants were asked to make a forced-choice judgment about which of two stimuli was upright, in a set of 8 pairs drawn from those used in this experiment (polarity $\times$ symmetry $\times$ colour). Twenty participants chose as upright all 8 stimuli in the wide-base orientation, one participant chose as upright 1 stimulus in the wide-top orientation, and one participant chose 3 stimuli in the widetop orientation as upright. 
2.1.4 Procedure. The participants were tested individually in a session that lasted approximately $40 \mathrm{~min}$. There was an initial practice block of 16 trials. When the participants felt at ease with the task, they started the experimental blocks, otherwise they would get another practice block of 16 trials.

A trial consisted of a fixation cross presented in the centre of the screen for $1000 \mathrm{~ms}$. After the fixation cross disappeared, the stimulus was presented until the participant responded or $15000 \mathrm{~ms}$ had passed. After the response, there was an intertrial interval of $1000 \mathrm{~ms}$, before a new trial would start.

The task of the participants was to look for a vertically symmetric object on the screen. If the target was present, participants had to push the present key on the keyboard as quickly as possible. If it was not there, they had to push the absent key. The present and absent keys were the ' $\mathrm{Z}$ ' and the ' $\mathrm{M}$ ', respectively, on a standard British keyboard. The assignment of the keys to either present or absent depended on the preferred hand of the participant: half pressed present with their preferred hand, half with their non-preferred hand.

2.1.5 Design. There were three within-subjects factors: polarity (wide base, wide top), target presence (symmetric target present, symmetric target absent), and the colour of the stimuli (black, white). All the factors were fully crossed. In total, there were 640 trials. The colours were presented in separate blocks, resulting in two separate sub-experiments of 320 trials. The sub-experiments each contained 4 blocks of 80 trials. Between every block there was a self-paced break for the participant. If a participant made an error, the error trial was inserted somewhere in the sequence of remaining trials. Each error resulted in an extra trial. Because the block size was fixed at 80, any error would result in an extra block. The size of this block depended on the number of errors made by the participant. After the first sub-experiment was finished (4 blocks, plus the added block containing the retake trials), there was a new training sequence of 16 trials, to give the participants the opportunity to acquaint themselves with the new colour.

\subsection{Results}

The results are shown in figure 2. Responses greater or less than 2.0 SDs from the cell mean (polarity $\times$ target presence $\times$ colour) for each participant were rejected. This resulted in rejection of $4.5 \%$ of the data. The error analysis was performed on all of the remaining trials, whereas the reaction-time analysis only used the correct trials.
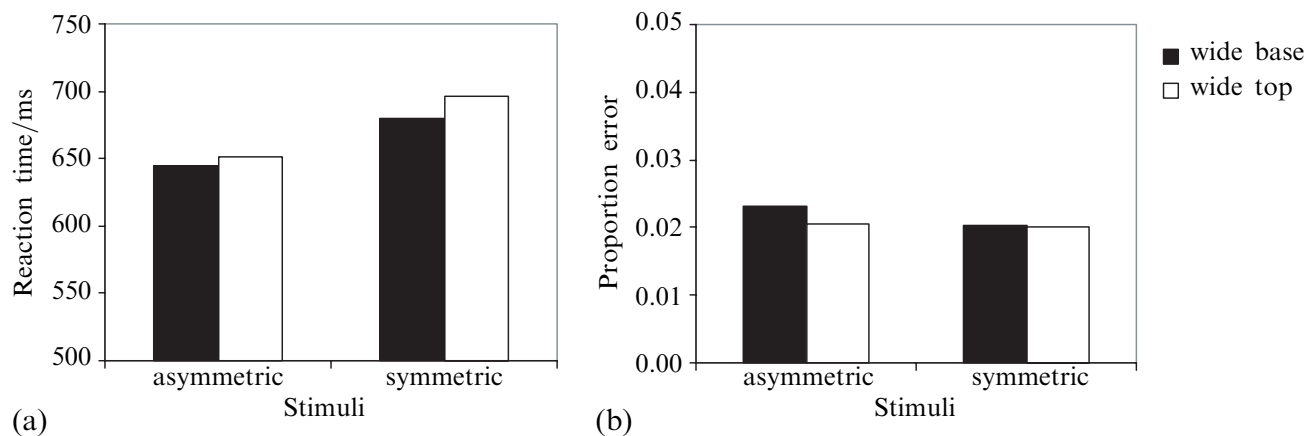

Figure 2. Results of experiment 1: (a) reaction times; (b) error proportions.

A three-way repeated-measures ANOVA (polarity $\times$ target presence $\times$ colour), yielded a significant main effect of polarity $\left(F_{1,9}=5.4, p<0.046\right)$. The effect of target presence approached significance $\left(F_{1,9}=4.4, p<0.066\right)$. None of the other main effects or interactions was significant $(p \mathrm{~s}>0.16)$.

A similar ANOVA on the error data showed no significant main effects, nor any significant interactions $(p \mathrm{~s}>0.2)$. 


\subsection{Discussion}

The results are clear-cut. There was an effect of inverting the polarity of the stimuli on the detection of symmetry. It took longer to decide whether a stimulus was vertically symmetric when it had a wide top than when it had a wide base. The effect of size per participant ranged from a $4 \mathrm{~ms}$ reaction-time advantage for wide-top stimuli to a $51 \mathrm{~ms}$ reaction-time advantage for wide-base stimuli. Nine of the ten participants showed an advantage for wide-base stimuli.

Even though our asymmetric stimuli did contain some symmetry at their outer ends (a factor that might influence the detection of symmetry-Bruce and Morgan 1975), this does not explain why there is an effect of polarity inversion, because the wide-base and the wide-top stimuli were inverted versions of each other. Instead of this, we suggest that top-bottom coding affected symmetry assignment. Symmetry judgments were faster to stimuli with a wide base and narrow top, that were more likely to be judged as upright, than their inverted partners (as indicated by the results from our rating study).

The effect of stimulus polarity (around $12 \mathrm{~ms}$ ) was certainly not large, possibly because of the ease of the basic discrimination task, which left little room for improvement; nevertheless, it was reliable. To the best of our knowledge, this is the first time that such an effect has been reported.

As indicated in section 1, this experiment was originally only intended as a control experiment for another series of studies. It therefore contains a confound between stimulus polarity and position. As shown in figure $1 \mathrm{~b}$, the wide-base and the widetop stimuli each appeared at a separate set of locations. Given the fact that a display contains only a single stimulus, which is bound to capture attention, we think that this confound is not serious and does not detract from our results. However, to ensure that the confound was not critical, in experiment 2 we presented both stimulus polarities at exactly the same four positions on the display.

\section{Experiment 2}

\subsection{Method}

Unless otherwise mentioned, the method was identical to that in experiment 1.

3.1.1 Participants. Fourteen new participants (eight female, six male) were recruited, either in return for course credit or for a small payment. All participants had normal or corrected-to-normal vision, and they were unaware of the purpose of this experiment.

\subsubsection{Apparatus. We used the same apparatus as in experiment 1.}

3.1.3 Stimuli. The stimuli were presented in black on a grey background covering the whole screen. The dimensions of the stimuli were identical to those in experiment 1 . The stimuli could appear in the centre of four virtual cells $3.7 \mathrm{deg}$ high and $4.5 \mathrm{deg}$ wide. The cells were part of a virtual bar $(3.7 \mathrm{deg} \times 17.9 \mathrm{deg})$, centred on the screen.

3.1.4 Procedure. The individual sessions for experiment 2 took about $20 \mathrm{~min}$. The rest of the procedure was identical to the one in experiment 1.

3.1.5 Design. In experiment 2, we dropped the colour manipulation. This leaves two within-subjects factors: polarity (wide base, wide top) and target presence (symmetric target present, symmetric target absent). Both factors were fully crossed. In total, there were 384 trials, subdivided into blocks of 96 . Between every block there was a self-paced break for the participant. Again, error trials were retaken.

\subsection{Results and discussion}

The results are shown in figure 3. Responses greater or less than $2.0 \mathrm{SDs}$ from the cell mean (polarity $\times$ target presence) for each participant were rejected. This time $4.4 \%$ 


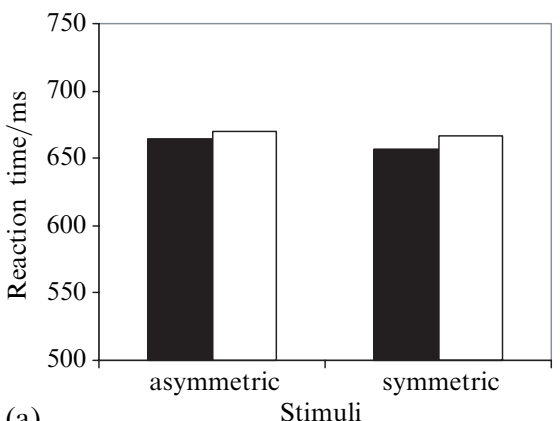

(a)

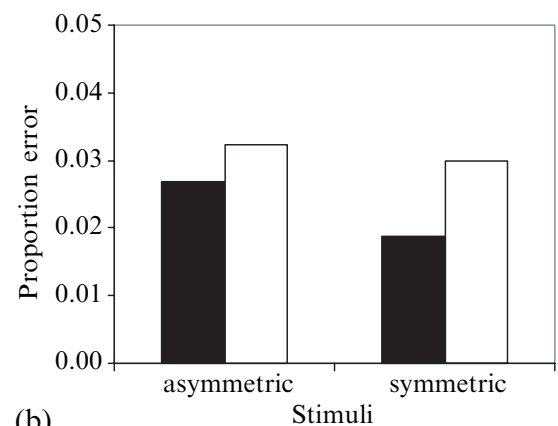

(b)

- wide base $\square$ wide top

Figure 3. Results of experiment 2: (a) reaction times; (b) error proportions.

of the data was rejected. The error analysis was performed on all of the remaining trials, whereas the reaction-time analysis used only the correct trials.

A two-way repeated-measures ANOVA on the reaction times (polarity $\times$ target presence), yielded a significant main effect of polarity $\left(F_{1,13}=6.9, p<0.021\right)$. There was no significant effect of target presence, and no interaction $(p s>0.5)$.

A similar ANOVA on the error data showed no significant main effects for target present, nor a significant interaction $(p \mathrm{~s}>0.4)$. The main effect of polarity approached significance $\left(F_{1,13}=4.1, p<0.065\right)$.

In experiment 2, we see a clear replication of the results of experiment 1. Again, there was an effect of inverting the polarity of the stimuli on the detection of symmetry. The effect was about the same size as the effect in experiment 1 , around $10 \mathrm{~ms}$. There was no sign of speed-accuracy tradeoff, and the error rates precisely followed the reaction-time results. For the individual participants, the effect sizes per participant ranged from a $7 \mathrm{~ms}$ reaction-time advantage for wide-top stimuli to a $28 \mathrm{~ms}$ advantage for wide-base stimuli. Ten of the fourteen participants showed an advantage for the wide-base stimuli.

\section{General discussion}

We have demonstrated a clear, albeit small, influence of stimulus polarity on the perception of vertical (bilateral) symmetry. Symmetry judgments are easier when stimuli have a wide base and a narrow top, relative to when they have a narrow base and a wide top.

Although previous studies have demonstrated that the efficiency of symmetry judgments is affected by the orientation of the main axis in the shapes (eg Palmer and Hemenway 1978; Royer 1981), and by the presence of axes of elongation (Sekuler and Swimmer 2000), none has previously shown that performance is influenced by whether the stimuli have a wide or a narrow base. There are at least two reasons for this. First, as far as we know, nobody has tried to manipulate the top-bottom polarity of vertically symmetric items before. The main focus in symmetry research has been on differences between orientations and on differences between the constituting elements of vertically symmetric items. These differences have typically been studied with the use of randomly generated pattern halves that were subsequently mirrored, precluding any systematic manipulation of top-bottom polarity. Second, even though the effect is consistent, it is rather small. We have had to use a considerable number of observations per cell to unearth it, in dedicated experiments. Other experiments might simply have lacked the power to detect the effect.

An important question remains: why should the detection of vertical symmetry be affected by polarity in the first place? An answer to this question might come from a consideration of more general constraints on visual processing. 
From the studies of Rock (1973) onward, it has been known that human vision is strongly affected by the assignment of top and bottom codes to stimuli, and it is well known that top-bottom inversion disrupts the identification of familiar objects (Jolicoeur 1985; Yin 1969). The stimuli we used in our experiments have a clear differentiation between their top part and their bottom part. Judging from our results, the top and bottom parts of the wide-base version of our stimuli are more compatible with the assignment of top and bottom codes than those of the wide-top version. This is suggested both by the two reaction-time experiments and the pilot experiment where introspective judgments were required. It is important to realise that there is no a priori reason to argue that the narrow end of the stimuli should be considered the top. The stimuli are just what they are: abstract shapes. Nevertheless, the results do support the conclusion that it is easier to assign 'top' to the narrow end of our stimuli than to the wide end.

The effect of inversion in object recognition is usually much larger than the inversion effect we report here. In our experiments, the task of the participants was symmetry detection, rather than object recognition, and therefore we should not expect an effect of the same size, even if the same processes are at play.

The most important conclusion to be drawn from our data is that symmetry perception has to conform to processes that are used for visual perception in general. Of course, after a little thought, this is perfectly natural: the visual system evolved for general purposes, not for symmetry perception in particular. Our data suggest that, in a symmetry detection task, participants make a decision about the top and the bottom of a stimulus, while they are looking for its axis of symmetry.

Acknowledgment. This work was supported by the UK Medical Research Council.

\section{References}

Baylis G C, Driver J, 1994 "Parallel computation of symmetry but not repetition within single visual shapes" Visual Cognition $1377-401$

Bruce V G, Morgan M J, 1975 "Violations of symmetry and repetition in visual patterns" Perception $4239-249$

Dakin S C, Watt R J, 1994 "Detection of bilateral symmetry using spatial filters". Special issue: The perception of symmetry. Part I. Theoretical aspects Spatial Vision $8393-413$

Jolicoeur P, 1985 “The time to name disoriented natural objects" Memory \& Cognition 13 289-303

Olivers C N L, Van der Helm P A, 1998 "Symmetry and selective attention: A dissociation between effortless perception and serial search" Perception \& Psychophysics $601101-1116$

Osorio D, 1996 "Symmetry detection by categorization of spatial phase, a model" Proceedings of the Royal Society of London, Series B $263105-110$

Palmer S E, Hemenway K, 1978 "Orientation and symmetry: Effects of multiple, rotational and near symmetries" Journal of Experimental Psychology: Human Perception and Performance 4 $691-702$

Rock I, 1973 Orientation and Form (New York: Academic Press)

Royer F L, 1981 "Detection of symmetry" Journal of Experimental Psychology: Human Perception and Performance $71186-1210$

Sekuler A B, Swimmer M B, 2000 "Interactions between symmetry and elongation in determining reference frames for object perception" Canadian Journal of Experimental Psychology 54 42-55

Wagemans J, 1995 "Detection of visual symmetries" Spatial Vision 9 9-32

Yin R K, 1969 "Looking at upside-down faces" Journal of Experimental Psychology 81 141-145 


\section{PERCEPTION}

VOLUME 332004

www.perceptionweb.com

Conditions of use. This article may be downloaded from the Perception website for personal research by members of subscribing organisations. Authors are entitled to distribute their own article (in printed form or by e-mail) to up to 50 people. This PDF may not be placed on any website (or other online distribution system) without permission of the publisher. 